\title{
Inter-varieties variation of sugar metabolism that inhibit photosynthesis: hexokinase-dependent stomatal and non-stomatal behaviors
}

\author{
Ruihua Ren ${ }^{1}$, Junnan Li $^{1}$, Jun Yang ${ }^{1}$, Lin Yuan ${ }^{1}$, Huangzhao Chen ${ }^{1}$, Huawei Chen ${ }^{1}$, \\ Pengfei Ning ${ }^{2}$, and Zhenwen Zhang ${ }^{1}$ \\ ${ }^{1}$ Northwest A\&F University \\ ${ }^{2}$ Affiliation not available
}

September 5, 2020

\begin{abstract}
The generation of sugar from photosynthesis and its utilization by sugar metabolism jointly determine the leaf sugar status, which conversely represses photosynthesis. The objective of this study was to investigate the effect of leaf sugar metabolism on photosynthesis in two contrasting grape (Vitis vinifera L.) genotypes, Riesling (RI, high level hexose) and Petit Manseng (PM, low level hexose). The total hexose and glucose contents of the RI leaves were significantly higher than those of PM relative to the low level of fructose. The activity of cell wall invertase for sucrose hydrolysis and the abundances of VvCWINV, VvHT4, VvTMT1, VvFK1 and VvFK1 transcripts were higher in RI leaves. The abundance of hexokinase transcript (VvHXK2) for signal sensing was also higher, while VvHXK1 was lower for glucose phosphorylation. The net photosynthetic rate, stomatal conductance, transpiration rate and chlorophyll content of RI leaves were lower than those of PM and negatively correlated with the hexose and glucose contents and the VvHXK2, VvCWINV, VvHT4, and VvTMT1 transcript levels. These results suggest that variation in sugar metabolism by inter-varieties alters the cellular sugar status, which is important for regulating photosynthesis. This study provides insights on improving the photosynthesis of cultivars by metabolic modification for breeding.
\end{abstract}

\section{Hosted file}

1.Manuscript.doc available at https://authorea.com/users/356478/articles/479342-intervarieties-variation-of-sugar-metabolism-that-inhibit-photosynthesis-hexokinasedependent-stomatal-and-non-stomatal-behaviors

\section{Hosted file}

3.Figs.doc available at https://authorea.com/users/356478/articles/479342-inter-varietiesvariation-of-sugar-metabolism-that-inhibit-photosynthesis-hexokinase-dependent-stomataland-non-stomatal-behaviors 